\title{
IMPROVING THE SPECIFIC COMPETENCES OF MASTER STUDENTS IN BREEDING SCIENCE: INTRODUCTION OF A PRACTICAL LESSON FOR ASSESSING BIOAVAILABILITY OF TARGET MOLECULES
}

\author{
C. Guijarro-Real, J. Prohens, A. Rodríguez-Burruezo, A. Fita \\ Universitat Politècnica de València, Instituto de Conservación y Mejora de la Agordiversidad \\ Valenciana (COMAV) (SPAIN)
}

\begin{abstract}
Modern consumers concern about the influence of diet in maintaining the health status. As consequence, Plant Breeding has included the enhancement of nutritional quality as a new goal in many programs. However, not all the bioactive molecules are equally stable, and the degradation by human body and bioavailability of different molecules can differ greatly. In this sense, understanding of chemistry and degradation of different nutritional compounds can become essential to address this issue, as a guide to increase efforts in the enhancement of the most stable target molecules. Thus, professionals must be able to develop and perform specific nutritional analyses in their breeding programs. Master studies are conceived as specialization studies for post-graduates, addressed to increase the knowledge and experience of future professionals. Thus, design of the educational programs should be such as to provide students with the specific competences needed during their careers. Our Institute offers a Master in Plant Breeding that includes the acquisition of specific competences by means of the development of practical lessons. Practical lessons are of paramount importance to understand and fix the knowledge exposed during the theoretical lessons, and are also conceived as a tool for students to gain experience and deal with real problems in their professional careers. As part of our educational program, we propose in this paper the introduction of a new practical lesson in the subject "Breeding for quality": the simulation of a digestion process in vitro for the analysis of bioavailability of target biomolecules. The lesson is designed to be developed during three sessions, in the course of which students will perform all the steps for this type of analyses. Steps include the preparation of samples and analysis of material prior to the digestion, the simulation of the digestion process in vitro, and the analysis of the digested material and evaluation of results. The lesson is designed to be performed by pairs, since we consider that this methodology facilitates the learning process, using different fruits and vegetables. In addition, during the last part of the lesson, students will be asked to share the experience and results with the class. Students' explanations will have special focus on any problems derived from the treatment of matrix and target molecules, allowing the discussion of these problems and possible solutions provided by students and educator. Finally, a one-minute test will be included in order to evaluate the acquisition of knowledge related to the key aspects of the lesson. At the end of the lesson, students should be able to perform an analysis of the bioavailability of different target biomolecules after a simulated digestion in vitro. The aim of this lesson is to understand that different bioactive compounds suffer different degradation grades. This knowledge will help future professionals to select the most promising and stable target molecules for starting breeding and food nutrition programs focused to enhance nutritional quality. Moreover, the lesson can be easily adapted to other Master studies related to Food Technology or even Human health studies, increasing specific competences for students in these fields as well.
\end{abstract}

Keywords: Bioavailability, compounds degradation, digestion, Plant science studies, practical lesson, specific competence.

\section{INTRODUCTION}

Master studies prepare postgraduates for a high level of expertise, with an advanced training for professional, academic and research specialization [1]. In order to reach such level of expertise, a strategy commonly followed is the integration of theoretical classes and practical lessons. In the particular case of Plant Science studies, practical lessons can become essential for the learning process and training. Thus, empirical evidence, experimented during these lessons, is considered one component of scientific knowledge, leading to a better understanding of the theoretical concepts [2]. 
On the other hand, future professionals should be able to put into practice what they learned before [3], and in these terms laboratory and field work allow students gaining these competences and skills.

Our Institute offers a Master in Plant Breeding, which has special focus in the development of new crop varieties for stress tolerance and for enhanced quality. With the goal of training future professionals with great competences and skills needed during their careers, we have designed an educational program in which laboratory and field works are strongly promoted. As part of our educational program, we propose in this paper the introduction of a new practical lesson in the subject "Breeding for quality": the simulation of a digestion process in vitro for the analysis of bioavailability of target biomolecules selected in breeding programs. The subject is addressed to teach and train students in the development of new crop varieties with an enhanced added value. In recent times, an increasing concern about the influence of diet in human health has appeared. In a scientific level, it has derived in a number of reports focused on this topic (e.g., [4], [5]). In addition, this concern has affected as well the position of consumers about diet, deriving in an increasing demand of food rich in bioactive compounds [6]. As a consequence, new opportunities in Plant Breeding have appeared, promoting the enhancement of nutritional quality as a new goal in many breeding programs. However, not all the bioactive molecules are equally stable, and their degradation by human body and bioavailability differ [7]. Thus, understanding the chemistry and degradation of different nutritional compounds can become essential as a guide to increase breeding efforts in the enhancement of the most stable target molecules, considering that only the fraction potentially bioavailable will be responsible to the real nutritional quality of the studied matrix [8].

We hypothesize that this practical lesson will help students to understand the importance that the stability of target molecules has in their bioavailability for human body, thus reinforcing the theoretical knowledge. This specific competence will allow future professionals to focus breeding programs on those molecules of greater interest, giving them the tools for performing previous analyses in order to decide the proper target molecules. In addition, the lesson is designed for enhancing other skills in students, such as working in pairs, managing of time and successful communication.

\section{PRACTICAL LESSON}

The practical lesson proposed in this paper is conceived to be included in the program of the subject "Breeding for quality". The design and methodology followed during this practical lesson mainly attends to the enhancement of a 'peer learning process' for a better fixation of knowledge by students. Peer learning is considered as a learning process that involve the sharing of knowledge, ideas and experience between students [9], as a tool to enhance the learning process. Thus, students will work by pairs during all lesson, with a total of ten groups formed, and at the end of the sessions, they will share experiences with the rest of groups.

The practical lesson is designed to be performed in three sessions, in the course of which students will perform all the steps for the development of an in vitro digestion process and analysis of results. As plant material, different fruits and vegetables are provided to students, previously selected by the instructor according to the content in target molecules and special characteristics of the molecules in the correspondent food matrixes. At the beginning of the lesson, the instructor provides a guide to students that includes the outline of the lesson (Fig. 1) and the protocols needed, and takes the first five minutes to explain the steps that students must perform. 
Day 1

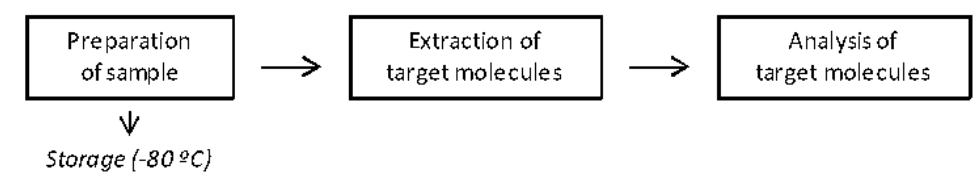

Day 2
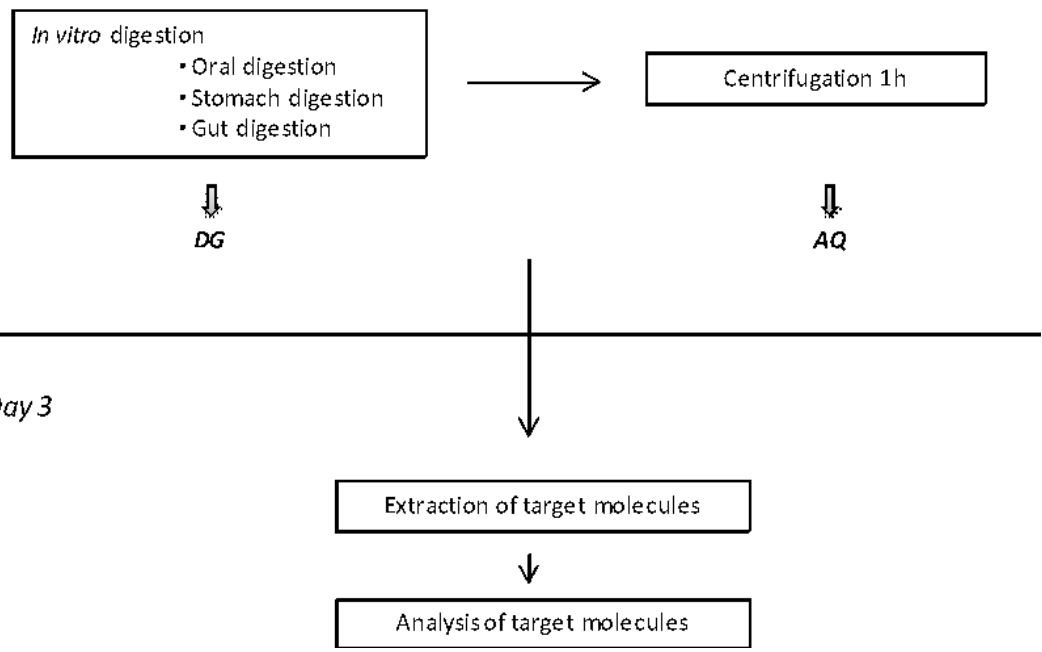

Figure 1. Scheme of the practical lesson. DG and AQ indicate the digestion product and aqueous fraction, respectively.

\subsection{Session one}

The first session is destined to the preparation of the samples that will be used during the lesson, and the analysis of the target molecules in the crude material, it is, before the digestion process. The session is organized to be completed in three hours, and developed as described in the following steps:

1 Homogenize the crude material with a commercial blender until an adequate puree is obtained. Depending on the fruit or vegetable used, students are asked to weigh a specific quantity before homogenize, and note the quantity weighed.

2 Obtain two adequate aliquots for the extraction of ascorbic acid and phenolics as target molecules, and store the puree extra at $-80^{\circ} \mathrm{C}$.

3 Use one aliquot for the extraction of ascorbic acid according to the protocol provided in the guide [10]. Filter the supernatant collected after centrifugation and prepare for analysis using the high pressure liquid chromatography (HPLC) technique. Once the samples are prepared, the instructor is the responsible in performing the HPLC analysis.

4 Use the second aliquot for the extraction of phenolic compounds according to the protocol provided [11]. Analyze the reducing capacity of total phenolics extracted using the FolinCiocalteu procedure [12], according to the protocol in the guide [11].

During the extraction and incubation times for phenolic compounds, students are asked to calculate the weigh and volumes needed for the proper preparation of the solutions needed, and for the theoretical preparation of the standard curve (Table 1). These calculations are essential for applying the correspondent protocols successfully. However, our experience indicates that many students have difficulties in this point, fact that can compromise the analyses. Thus, training students in the preparation of solutions and standards enhance the preparation of future professionals. Nevertheless, for the practice all solutions needed have been previously prepared by the instructor, and directly provided to the students. 
Table 1. Example of the questionnaire that students are asked to fill during the first session.

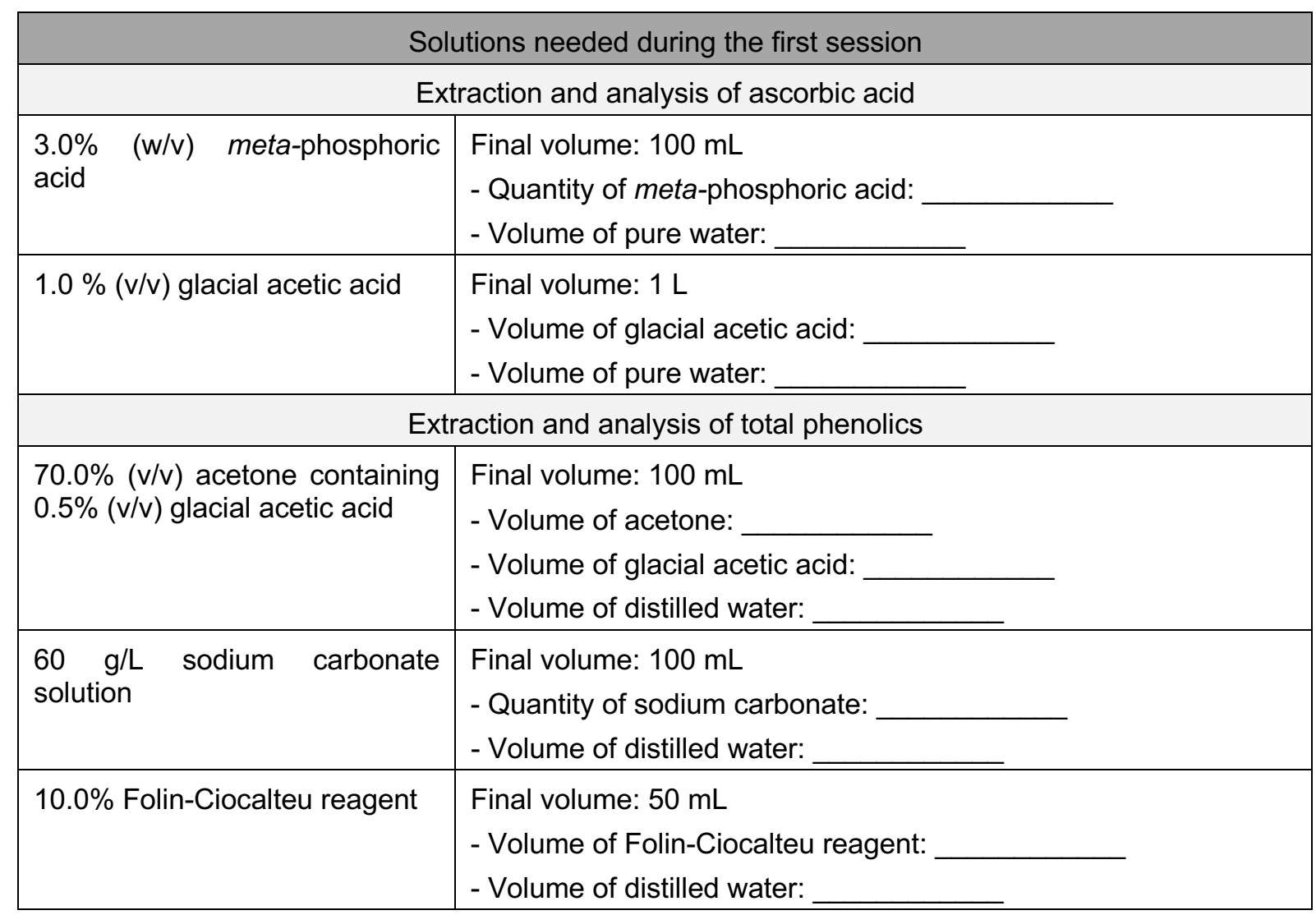

At the end of the first session, students must know the content in ascorbic acid and total phenolics in the crude samples analyzed. These data are needed for the final results of the lesson, so they must store the results.

\subsection{Session two}

The second session is destined to simulate an in vitro digestion process and consequent hydrolysis and degradation of target molecules by the enzymes action. Students are asked to follow the protocol adapted from [13]. Due to the long incubation times, the session is designed to be performed in three hours by students, while the instructor will perform the final step after gut incubation and storage of samples. As in session one, the instructor will provide students with the solutions needed.

The steps to perform are the following:

1 Weigh a proper quantity of unfrozen sample, homogenize with the oral phase solution and incubate (Fig. 2a). In order to accelerate the start of the session, the instructor will previously unfreeze all the samples.

2 Homogenize with the gastric phase solution in a proper $\mathrm{pH}$ and incubate (Fig. 2b). During this step, students need to adjust carefully the $\mathrm{pH}$ using a pH-meter (Fig. 2c). Thus, the instructor will previously provide a guideline for the use of this instrument and some tips for a quick and correct adjustment of $\mathrm{pH}$.

3 Homogenize with the intestinal phase solution in a proper $\mathrm{pH}$ and incubate. The incubation time of the intestinal phase is of two hours. Thus, the session is designed to be continued from this point on by the instructor. In order to ensure that the learning process is complete, the instructor will explain the remaining steps in a short video or alternatively as a sequence of photographs, as described as follow (steps 4 to 6 ).

4 Store an aliquot that represents the finished digestion product (DG), it is, the product after the digestion is concluded.

5 Centrifuge the remaining DG (Fig. 2d). 
6 Store an aliquot of the aqueous fraction (AQ), it is, the liquid that can be absorbed by the body.

A

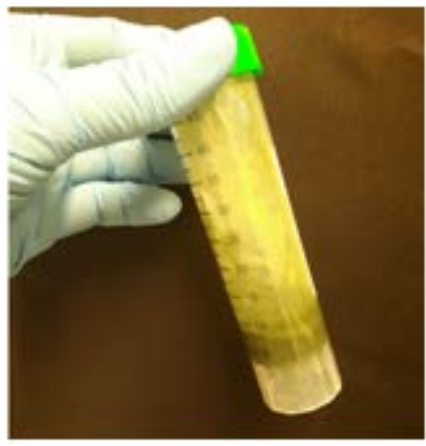

$\mathrm{C}$

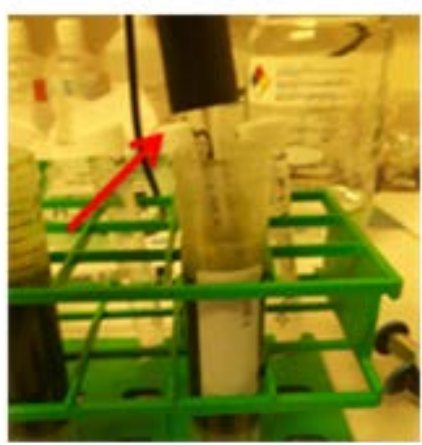

B

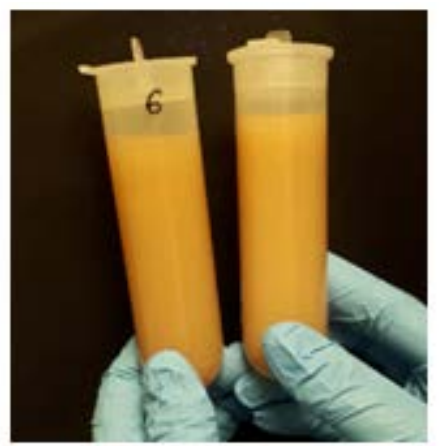

D

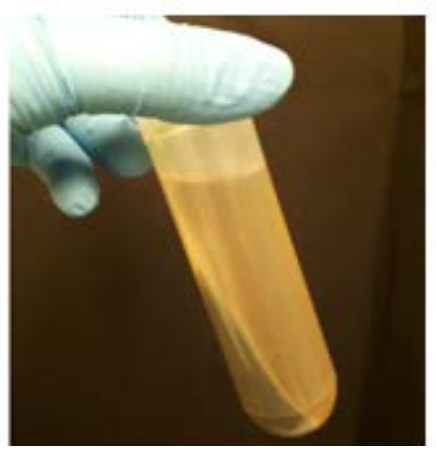

Figure 2. Example of the steps followed during the digestion protocol. 2A. Product of the oral phase incubation. 2B. Product of the gastric phase incubation. 2C. Use of $\mathrm{pH}$-meter for proper adjustment of $\mathrm{pH}$. $2 D$. Product of the centrifugation of digesta phase.

As in the first session, during the incubation times students are asked to provide the calculations for the theoretical preparation of the solutions needed (Table 2). Once all students have completed the task, the instructor will share the results with the class, so students can detect possible mistakes in their estimates.

Table 2. Example of the questionnaire that students are asked to fill during the second session.

\begin{tabular}{|l|l|}
\hline \multicolumn{2}{|c|}{ Solutions needed during the second session } \\
\hline $\begin{array}{l}\text { Oral phase solution containing } 3000 \\
\text { units of } \alpha \text {-amylase }\end{array}$ & Final volume: $10 \mathrm{~mL}$ \\
& - Quantity of $\alpha$-amylase: \\
& - Volume of base solution: \\
\hline $\begin{array}{l}\text { Gastric phase solution containing } 10 \\
\mathrm{mg} \mathrm{mL}^{-1} \text { of pepsin }\end{array}$ & Final volume: $10 \mathrm{~mL}$ \\
& - Quantity of pepsin: \\
\hline Intestinal phase solution 1 & - Volume of base solution: \\
containing $10 \mathrm{mg} \mathrm{mL}^{-1}$ of lipase and & Final volume: $10 \mathrm{~mL}$ \\
$20 \mathrm{mg} \mathrm{mL}^{-1}$ of pancreatin & - Quantity of lipase: \\
\hline Intestinal phase solution 2 & - Quantity of pancreatin: \\
containing $30 \mathrm{mg} \mathrm{mL}^{-1}$ of bile extract & - Volume of base solution: \\
\hline & Final volume: $10 \mathrm{~mL}$ \\
\hline
\end{tabular}

\subsection{Session three}

The last session, of three hours, is destined to analyze the content in target molecules after the digestion process. Thus, students will follow the same protocols as in session 1 for the extraction and analysis of ascorbic acid and total phenolics, from the DG and AQ aliquots. Results from the first and 
third session will be then used for estimating 1 ) the stability of the target molecules during the digestion, and 2) the micellarization efficiency.

The last part of this session is used for the analysis of the results obtained. If the protocol has been successfully applied, students will find that ascorbic acid and phenolic compounds have different digestion stabilities. Then, guided by the instructor, students will try to explain the reasons for this different behaviour. In addition, the instructor will use this moment to draw different problems that can occur during the adaptation of the protocol to different matrixes, so students will have the opportunity to discuss and obtain possible solutions. Finally, students will answer a one-minute test used as a tool to evaluate the knowledge acquired (Table 3 ).

Table 3. Example of questions for the one-minute test used to evaluate the knowledge acquired.

\section{One-minute test}

1. Why does the digestion process apparently increase the content in total phenolics? Which analytical technique could I use in order to unequivocally study the content variations during the digestion process?

2. Why is it possible to find high micellarization efficiencies for the target molecules? Would we obtain similar results with less hydrophilic molecules such as carotenoids?

3. I want to study the content in a target molecule after each digestion step (oral cavity, stomach and gut). For that reason, I take one aliquot after each digestion step and continue the protocol with the remaining product. Is it the proper way to work? What should I do instead?

\section{CONCLUSIONS}

This practical lesson should provide students with the specific competence of analyzing the content variation of target molecules associated to the digestion process. Students are asked to evaluate two different target molecules that have a different degradation response to the digestion. Thus, the aim of the lesson is to reinforce the theoretical knowledge of the stability that different biocompounds possess. This knowledge will help future professionals to focus breeding efforts on the proper molecules for a real enhancement of the nutritional aspects of fruits and vegetables. At the end of the lesson, students should be able to adapt the protocol to different food matrixes and molecules of interest. The discussion of the possible problems occurred during the lesson, together with other ones suggested by the instructor, will prepare students to successfully solve them in the future. In addition, the lesson is used as well as training for the correct preparation of solutions, a weakness detected in many students. Finally, this practical lesson can be adapted to other Master studies in Plant Science, Food Technology, Nutrition and Human health, increasing specific competences for students in these fields as well.

\section{ACKNOWLEDGEMENTS}

Carla Guijarro-Real is grateful to the Ministerio de Educación, Cultura y Deporte of Spain for financial support by means of a predoctoral FPU grant (FPU14-06798). 


\section{REFERENCES}

[1] I. Andújar, S. Vilanova, A. Fita, M. Plazas, A. Rodríguez-Burruezo, P. Gramazio, S. Soler, F.J. Herraiz, D. Borrás, M.R. Figàs, E. Moreno, M.D. Lerma, J. Prohens, "Development and introduction of a high technology practical session for the improvement of analysis techniques for Master students in Plant Sciences", Proceedings of INTED2015 Conference, pp. 288-294, 2015.

[2] R. Havdala, G. Ashkenazi, "Coordination of theory and evidence: Effect of epistemological theories on students' laboratory practice", Journal of Research in Science Teaching, 44, pp. 11341159, 2007.

[3] J. Wrenn, B. Wrenn, "Enhancing learning by integrating theory and practice", International Journal of Teaching and Learning in Higher Education, 21, pp. 258-265, 2009.

[4] H.D. Woo, J. Lee, I.J. Choi, C.G. Kim, J.Y. Lee, O. Kwon, J. Kim, "Dietary flavonoids and gastric cancer risk in a Korean population", Nutrients, 6, pp. 4961-4973, 2014.

[5] C. Zhang, L. Shu, H. Kim, T.O. Khor, R. Wu, W. Li, A.T. Kong, "Phenethyl isothiocyanate (PEITC) suppresses prostate cancer cell invasion epigenetically through regulating microRNA-194", Molecular Nutrition and Food Research, 60, pp. 1427-1436, 2016.

[6] J. Olayanju, "Perspectives on factors driving new trends in the food and drink", Forbes, Accessed $6^{\text {th }}$ February 2019. Retrieved from https://www.forbes.com/sites/juliabolayanju/2018/10/01/ perspectives-on-factors-driving-new-trends-in-the-food-drink/

[7] J.I. Mosele, A. Macià, M.P. Romero, M.J. Motilva, "Stability and metabolism of Arbutus unedo bioactive compounds (phenolics and antioxidants) under in vitro digestion and colonic fermentation", Food Chemistry, 201, pp. 120-130, 2016.

[8] H. Sengul, E. Surek, D. Nilufer-Erdil, "Inverstigating the effects of food matrix and food components on bioaccessibility of pomegranate (Punica granatum) phenolics and anthocyanins using an in-vitro gastrointestinal digestion model", Food Research International 62, pp. 10691079, 2014.

[9] D. Boud, "Introduction: making the move to peer learning" in Peer learning in higher education: learning from and with each other (D. Boud, R. Cohen and J. Sampson eds.), pp. 1-20, New York: Routledge, 2013.

[10] A. Cano, A. Bermejo, "Influence of rootstock and cultivar on bioactive compounds in citrus peels", Journal of the Science of Food and Agriculture, 91, pp. 1702-1711, 2011.

[11] M.D. Raigón, J. Prohens, J.E. Muñoz-Falcón, F. Nuez, "Comparison of eggplant landraces and commercial varieties for fruit content of phenolics, minerals, dry matter and protein", Journal of Food Composition and Analysis, 21, pp. 370-376, 2008.

[12] V.L. Singleton, J.A. Rossi, "Colorimetry of total phenolics with phosphomolybdic phosphotungstic acid reagents", American Journal of Enology and Viticulture, 16, pp. 144-158, 1965.

[13] N. Girgin, S.N. El, "Effects of cooking on in vitro sinigrin bioaccessibility, total phenols, antioxidant and antimutagenic activity of cauliflower (Brassica oleraceae L. var. botrytis)" Journal of Food Composition and Analysis, 37, pp. 119-127, 2015. 